\title{
EXAMPLES OF $p$-ADIC TRANSFORMATION GROUPS
}

BY FRANK RAYMOND AND R. F. WILLIAMS ${ }^{1}$

Communicated by Deane Montgomery, June 11, 1960

1. Introduction. Our purpose here is to outline the construction of an $n$-dimensional space $X^{n}, n \geqq 2$, upon which the $p$-adic group $A_{p}$ acts so that the orbit space $X^{n} / A_{p}$ is of dimension $n+2$. Though such examples are new, it had been known $[1 ; 4]$, that either they do exist or a certain long standing conjecture on transformation groups must be true. The conjecture states that every compact effective group acting on a (generalized) manifold must be a Lie group; it may well be false.

Another question concerns the amount, $k$, by which the (cohomology) dimension of a compact space can be raised under the decomposition map $X \rightarrow X / A_{p}$. By $[1 ; 4], k \leqq 3$. (An example in which $k=1$ is essentially due to Kolmogoroff [2].) No example is known for which $k=3$. The authors expect to have more to say on this subject.

2. The building blocks. There exists an $(n+2)$-dimensional complex $X_{i}$, a homeomorphism $h_{i}: X_{i} \rightarrow X_{i}$, maps $q_{i}: X_{i} \rightarrow s^{n+2}$ and $r_{i}: X_{\imath} \rightarrow X_{i}$ such that ( $S^{n+2}$ is the standard $n+2$-simplex)

(a) $h_{i}$ is of period $p^{i}$.

(b) $q_{i}^{-1}(j$ skeleton $)=X_{i}(j)$ is a $j$-complex, $j=0,1, \cdots, n+2$.

(c) $q_{i} h_{i}=q_{i}$ so that $q_{i}$ can be factored through the orbit space: $i=1, p, \cdots, p^{i-1}$

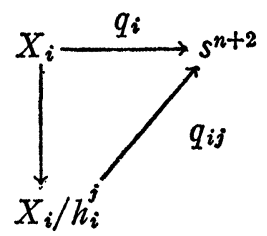

(d) $q_{i j^{*}}: H_{n+2}\left(X_{i} / h_{i}^{j}, \quad X_{i}(n+1) / h_{i}^{j} ; \quad Z_{p}\right) \rightarrow H_{n+2}\left(s^{n+2}, s^{n+2} ; Z_{p}\right)$ is onto; and

(e) $r_{i}: X_{i} \rightarrow X_{i}(n)$ is a retraction.

3. $(E, \pi, B, X, q)$. Let $m$ be a positive integer and $s_{0}$ be the standard $m$-simplex. We consider 5 -tuples $(E, \pi, B, X, q)$, where $E, B$, and $X$ are $m$-complexes and $\pi, q$ are simplical maps such that

A1. $\pi: E \rightarrow B, q: X \rightarrow s_{0}$,

A2. for each $m$-simplex $s \in B$, there are specified maps

\footnotetext{
1 Both authors are National Science Foundation Fellows.
} 


$$
\begin{aligned}
& \psi_{s}: X \rightarrow \pi^{-1}(s)=E_{8}, \\
& \phi_{s}: s_{0} \rightarrow s
\end{aligned}
$$

where $\phi_{s}$ is a linear homeomorphism;

A3. commutativity holds in the diagram

$$
\begin{aligned}
& X \stackrel{\psi_{s}}{\rightarrow} E_{s} \\
& q \downarrow \underset{\downarrow}{s_{0}} \underset{\phi_{s}}{\rightarrow} s
\end{aligned}
$$

A4. For $s, s^{\prime}$ two $m$-simplexes of $K$,

$$
\psi_{s}\left|X_{s} \cap_{s^{\prime}}=\psi_{s^{\prime}}\right| X_{s} \cap_{s^{\prime}}, \text { and } \phi_{s}^{-1}\left|s \cap s^{\prime}=\phi_{s^{\prime}}^{-1}\right| s \cap s^{\prime},
$$

where $X_{s^{\prime} \cap_{s^{\prime}}}=q^{-1} \phi_{s}^{-1}\left(s \cap s^{\prime}\right)$.

We need two results concerning such 5-tuples.

3.1. Given $X, q$, and a complex $B$ which is the barycentric subdivision of a complex, there exists a complex $E$ and a map $\pi: E \rightarrow B$ such that $(E, \pi, B, q, X)$ satisfies A1-A4 and such that the $\psi_{s}^{\prime}$ 's are homeomorphisms.

3.2. If in addition to $\mathrm{A} 1-\mathrm{A} 4$, we assume

A5. $H_{m}\left(X, q^{-1}(\dot{s}) ; G\right) \rightarrow^{q *} H_{m}(s, \dot{s} ; G)$ is onto, then $H_{m}(E ; G)$ $\rightarrow H_{m}(B ; G)$ is onto.

4. The example. $X^{n}$ is defined as the inverse limit of a sequence

$$
E_{0} \leftarrow E_{\pi_{1}} \leftarrow E_{\pi_{2}} \leftarrow \cdots
$$

such that on each $E_{i}\left(\right.$ an $(n+2)$-complex) we have a map $\bar{h}_{i}$ of period $p^{i}$. The $E_{i}$ are defined inductively. $E_{0}$ is taken to be a triangulated $(n+2)$-sphere, and $\bar{h}_{0}=$ identity. Suppose $E_{i}, \pi_{i}, \bar{h}_{i}$ have been defined. Then set $B_{i+1}=$ the barycentric subdivision of $E_{i}$ and use 3.1 relative to $B_{i+1}, X_{i+1}, q_{i+1}$ (see $\S 2$ ), to obtain $E_{i+1}, \pi_{i+1}$. The homeomorphism $\bar{h}_{i+1}$ is defined on $E_{i+1}$ in terms of $h_{i+1}, \pi_{i+1}$ and $\bar{h}_{i}$, so that we have the equivariance

$$
\pi_{i+1} \bar{h}_{i+1}=\bar{h}_{i} \pi_{i+1}
$$

Thus the map

$$
\left(e_{0}, e_{1}, \cdots\right) \rightarrow\left(\bar{h}_{0} e_{0}, \bar{h}_{1} e_{1}, \cdots\right)
$$

defines an effective action of the $p$-adic group on $X^{n}$.

It is next shown that $\bar{E}_{i}=E_{i} / \bar{h}_{i}, i=1,2, \cdots$ satisfy axioms 
A1-A5 of $\S 3$, where $\bar{\pi}_{i}: E_{i} / \bar{h}_{i} \rightarrow E_{i-1} / \bar{h}_{i-1}$ is defined via (2c), and the $\psi_{s}$ 's are essentially like the $q_{i j}$ 's in (2c). By (3.2) all homeomorphisms in the sequence

$$
H_{n+2}\left(\bar{E}_{0} ; Z_{p}\right) \underset{\bar{\pi}_{1 *}}{\longleftarrow} H_{n+2}\left(\bar{E}_{1} ; Z_{p}\right) \leftarrow \cdots
$$

are onto so that the orbit space, $X / A_{p}$, which is the inverse limit of the sequence

$$
E_{0} / \bar{h}_{0} \underset{\bar{\pi}_{1}}{\leftarrow} E_{1} / \bar{h}_{0} \underset{\bar{\pi}_{2}}{\leftarrow} E_{2} / \bar{h}_{2} \underset{\bar{\pi}_{3}}{\leftarrow} \cdots
$$

is $(n+2)$-dimensional.

Finally, it follows from (2e) that $X^{n}$ is $n$-dimensional.

\section{BIBLIOGRAPHY}

1. G. E. Bredon, Frank Raymond and R. F. Williams, p-adic groups of transformations, to appear.

2. A. Kolmogoroff, Über offene Abbildungen, Ann. of Math. vol. 38 (1937) pp. 36-38.

3. D. Montgomery and L. Zippin, Topological transformation groups, New York, Interscience, 1955.

4. C. T. Yang, p-adic transformation groups, to appear.

INSTITUTE FOR AdVANCED STUdy 